\title{
Deciphering mycorrhizal fungi in cultivated Phalaenopsis microbiome with next-generation sequencing of multiple barcodes
}

\author{
Chao-Li Huang • Feng-Yin Jian • Hao-Jen Huang • \\ Wen-Chi Chang • Wen-Luan Wu • Chi-Chuan Hwang • \\ Ruey-Hua Lee • Tzen-Yuh Chiang
}

Received: 5 July 2013 / Accepted: 6 February 2014 / Published online: 25 February 2014

(C) The Author(s) 2014. This article is published with open access at Springerlink.com

\begin{abstract}
Identifying the species composition of a microbial ecosystem is often hampered by difficulties in culturing the organisms and in the low sequencing depth of traditional DNA barcoding. Metagenomic analysis, a huge-scale nucleotide-sequence-based tool, can overcome such difficulties. In this study, Sanger sequencing of 500 nrITS clones uncovered 29 taxa of 19 fungal genera, whereas metagenomics with next-generation sequencing identified 512 operational taxonomic units (OTUs) for ITS1/2 and 364 for ITS3/4. Nevertheless, high throughput sequencing of PCR amplicons of ITS1/2, ITS3/4, nrLSU-LR, nrLSU-U, mtLSU, and mtATP6, all with at least 1,300× coverage and about 21 million reads in total, yielded a very diverse fungal composition. The fact that $74 \%$ of the OTUs were exclusively uncovered with single barcodes indicated that each marker provided its own insights into the fungal flora. To deal with the high heterogeneity in the data and to integrate the information on species composition across barcodes, a rank-scoring strategy
\end{abstract}

Chao-Li Huang, Feng-Yin Jian, Hao-Jen Huang, Wen-Chi Chang and Wen-Luan Wu contributed equally.

Electronic supplementary material The online version of this article (doi:10.1007/s13225-014-0281-x) contains supplementary material, which is available to authorized users.

C.-L. Huang • F.-Y. Jian · H.-J. Huang $•$ W.-L. Wu •

T.-Y. Chiang $(\bowtie)$

Department of Life Sciences, National Cheng Kung University,

Tainan, Taiwan 701

e-mail: tychiang@mail.ncku.edu.tw

W.-C. Chang $\cdot$ R.-H. Lee $(\bowtie)$

Institute of Tropical Plant Sciences, National Cheng Kung

University, Tainan, Taiwan 701

e-mail: shanhua@mail.ncku.edu.tw

C.-C. Hwang $(\triangle)$

Department of Engineering Science and Supercomputing Research

Center, National Cheng Kung University, Tainan, Taiwan 701

e-mail: chchwang@mail.ncku.edu.tw was developed. Accordingly, 205 genera among 64 orders of fungi were identified in healthy Phalaenopsis roots. Of the barcodes utilized, ITS1/2, ITS3/4, and nrLSU-U were the most competent in uncovering the fungal diversity. These barcodes, though detecting different compositions likely due to primer preference, provided complementary and comprehensive power in deciphering the microbial diversity, especially in revealing rare species.

Keywords DNA barcode $\cdot$ Metagenomics $\cdot$ Mycorrhizal fungi $\cdot$ Next-generation sequencing $\cdot$ Phalaenopsis orchid . Rank scoring

\section{Introduction}

The Orchidaceae (orchids) is one of the largest families of angiosperms (Pridgeon et al. 2005). A great number of orchid species have been developed commercially as potted flowering crops with an annual market growth rate of $30 \%$ (Wang 2004). Among these, the monopodial epiphytic Phalaenopsis, one of the most popular orchids, is only available in the retail markets when in bloom. Over the past decades, a large pool of cultivars with new traits and phenotypic variation has been generated via traditional breeding.

Great advances in tissue culture techniques have also allowed mass production of disease-free orchid plantlets from seeds or vegetative tissues. One of the major problems in orchid production is that 1-year-old tissue-culture plantlets require at least 16-24 months of vegetative growth for the leaf span to reach a minimum diameter of $25 \mathrm{~cm}$ (Konow and Wang 2001; Runkle et al. 2007). The ability of Phalaenopsis to spike and bloom under inducive conditions, e.g., low temperatures, is highly correlated with the size of the plant; however, fungal infection can greatly reduce plant size. In addition, common pathogens such as Fusarium oxysporum 
(Beckman 1987), Sclerotium rolfsii (Cating et al. 2009), and Botrytis cinerea (Wey 1988) cause various unsightly symptoms on leaves and roots that, even if the orchid survives the disease, the quality and growth of orchids are irrevocably damaged and ruined for the commercial market.

Plant roots are surrounded by the rhizosphere, which contains compounds that are secreted by roots and microbes, which in turn influences the growth and survival of the organisms (Hinsinger et al. 2009). The interplay between plants and rhizosphere microorganisms can therefore affect plant growth and health (Bisseling et al. 2009; Berendsen et al. 2012). In return, photosynthetic plants secrete up to $21 \%$ of their fixed carbon to the rhizosphere as nutrients, feeding the microflora and influencing their metabolic activity and diversity (Mendes et al. 2011). For all or part of their life cycle, orchids are obligatorily dependent on their mycorrhizal partners in nature. For example, orchid seed is less likely to germinate in the absence of mycorrhizal fungi under natural conditions (Burgeff 1959), and orchid plants depend on the symbionts to gain access to organic and mineral nutrients by increasing nutrient absorption and translocation to plants via extraradical hyphae (Arditti 1992; Rasmussen 1995; Smith and Read 2008). Studying the microbiome of orchid roots enables one to understand the complexity of plant-microbe interactions associated with plant health and growth, thus opening new avenues to increase orchid quality and productivity.

Although scientists have traditionally depended on in vitro and in vivo culturing to explore fungal communities, most species remain unculturable, and rare strains can be easily unexploited in culture (Kaeberlein 2002). Technically optimizing culture conditions for individual species, especially when the species composition of a community remains unknown, can be time-consuming and difficult, especially to induce sporulation. In addition, direct observation of fungal morphotypes via isolation of a single peloton in roots requires expertise for accurate interpretation and is very timeconsuming. DNA barcodes, biochemical markers, and analysis of acyl chain composition in membrane-phospholipids also provide powerful tools for studying microbial ecology without conventional culture (Alef and Nannipieri 1995). Of these methods, DNA barcoding is a powerful tool to identify species using sequences for gene regions that are conserved across greatly diverse taxonomic groups (Hebert and Gregory 2005; Schoch et al. 2012). Nuclear ribosomal RNA (nrRNA) is the most abundant RNA encoded by ribosomal RNA (rRNA) genes. High conservation in the genes thus provides a framework for assigning sequences to genera and species for investigations of microbial community diversity (Rosselló-Mora and Amann 2001; Hirsch et al. 2010). Eukaryotic nrRNA barcodes include large subunit 28S rRNA (nrLSU) gene, small subunit 18S rRNA (nrSSU) gene, and the internal transcribed spacer (nrITS) rDNA plus the $5.85 \mathrm{~S}$ gene (Druzhinina et al. 2005; Kõljalg et al. 2005). Among these regions, nrITS is the most effective discriminator of fungal species, and the nrLSU is also very effective. The nrSSU, despite its prominence in assessing bacterial community, is too conserved for fungi (Tringe and Hugenholtz 2008; Schoch et al. 2012).

With the invention of next-generation sequencing (NGS), fungus-specific barcoding primers can be used with metagenomics, a huge-scale nucleotide-sequence-based tool, to analyze microbial communities regardless of an organism's culturability (Cowan et al. 2005). The tool provides high throughput sequencing of PCR amplicons from a single DNA extraction and estimates of the relative abundance of the organisms detected (Hirsch et al. 2010). However, because a single barcode is limited in representing the panorama of a microbial community, combinations of multiple barcodes have thus been recommended (DeSalle et al. 2008). Based on the evaluation of Schoch et al. (2012), we selected four nuclear ribosomal markers, two nrITS regions (ITS1/2 and ITS3/4) and two in the nrLSU region (nrLSU-LR and nrLSUU) (Vilgalys and Hester 1990; Wu et al. 2002). The large subunit of the mitochondria ribosomal region (mtLSU) and the sixth subunit of mitochondrial ATPase (mtATP6) (Zeng et al. 2004; Grubisha et al. 2012) have also been adopted as markers.

In this study, we deciphered the microbiome of cultivated orchid roots based on amplicon-based metagenomics. Using multiple barcodes, we investigated the taxon diversity of the fungal community and examined the consistency among barcodes in uncovering the composition of the fungal flora and the ecological interactions between fungal endophytes and orchids. We also compared traditional Sanger sequencing of full-length nrITS with NGS techniques. A rank-scoring strategy was also developed to integrate the information on species composition across barcodes.

\section{Materials and methods}

Plant materials and DNA extraction

Phalaenopsis KC1111 (Phalaenopsis Taisuco Snow $\times$ Doritaenopsis White Wonder) was obtained from the Taiwan Sugar Corporation (Taisuco) and grown in the greenhouse of National Cheng Kung University in Tainan, Taiwan. Plants were watered once a week without any pesticide or fertilizer. Microbial contamination from the potting media was eliminated by sterilizing the roots from five individuals of Phalaenopsis $\mathrm{KC} 1111$ in $2 \% \mathrm{NaOCl}$ for 15 min with five subsequent washes with water (Zelmer et al. 1996). These tissues were ground into powder with liquid nitrogen. Total genomic DNAs were extracted by using a modified cetyltrimethylammonium bromide (CTAB) method (Doyle and Doyle 1987). 
Gene cloning and Sanger sequencing

Full-length nrITS genomic DNA region, a marker often used for identifying fungi (Nilsson et al. 2008), was PCR amplified using the ITS1/ITS4 primer pairs (Wu et al. 2002) in a $50 \mu \mathrm{L}$ reaction mixture containing $25 \mu \mathrm{L}$ Taq DNA Polymerase $2 \times$ Master Mix Red (Ampliqon, Denmark), $5 \mu \mathrm{L}$ forward and reverse primers (ITS1 and ITS4, $2 \mathrm{ng} / \mu \mathrm{L}$, Table S1) each, and $5 \mu \mathrm{L}$ genomic DNA $(2 \mathrm{ng} / \mu \mathrm{L})$. The PCR cycling scheme consisted of one cycle of $94^{\circ} \mathrm{C} / 3 \mathrm{~min} ; 35$ cycles of $94^{\circ} \mathrm{C} / 30 \mathrm{~s}$, $55^{\circ} \mathrm{C} / 37 \mathrm{~s}, 72{ }^{\circ} \mathrm{C} / 30 \mathrm{~s}$; and a final extension at $72{ }^{\circ} \mathrm{C} / 10 \mathrm{~min}$. PCR products of $\sim 650 \mathrm{bp}$ were purified with a Gel/PCR DNA Fragment Extraction Kit (Geneaid, Taiwan), ligated into the pGEM-T Easy Vector (Promega, USA), and used to transform DH5 $\alpha$ competent cells (Yeastern Biotech, Taiwan). Clones were sequenced with an ABI PRISM 3730 DNA Sequencer (ABI Big Dye Terminator Cycle Sequencing Kit, PerkinElmer). The obtained sequences were used in a BLAST search against the NCBI (http://blast.ncbi.nlm.nih.gov/Blast.cgi) database with default blastn settings and assigned to specific taxa using MEtaGenome Analyzer (MEGAN) software (Huson et al. 2011). With MEGAN software, the lowest common ancestor (LCA) algorithm was used for taxonomic classification, with the required parameters of the LCA assignment set as minimum support $=1$, minimum score $=500$, top percentage $=1$.

Metagenomic barcoding of the fungal community in orchid roots

Six DNA fragments derived from four DNA regions, namely, nrITS (ITS1/2 and ITS3/4), nrLSU (LR and U), mitochondrial large subunit rDNA (mtLSU), and mitochondrial ATPase subunit 6 (mtATP6), were PCR-amplified using genomic DNA isolated from roots of cultivated Phalaenopsis KC1111. PCR primers and annealing temperatures are listed in Table S1. Amplification was conducted as described in the gene cloning section. All PCR products of ca. 250-300 bp were purified, pooled, and sequenced with Illumina GAIIx high-throughput paired-end sequencing to survey the composition of fungal community. Raw reads were sorted into six categories according to the primer sequences, and the reads with an $\mathrm{N}$ residue in the sequences were discarded. Sorted sequences were merged to haplotypes for computing the copy numbers, and single-copy haplotypes were removed to lessen the effect of sequencing errors. These haplotypes were further clustered into operational taxonomic units (OTUs) using the BLASTClust program in the standalone BLAST v2.2.26 package of the NCBI. Because the average minimal divergence between fungal species is around 2.5-3\% (Seena et al. 2010; Stockinger et al. 2010), the stringency of clustering was set with two parameters at $97 \%$ similarity and $80 \%$ coverage between sequences and referred to as the average minimal divergence of species between fungi. From reads sorting, singleton removal, to OTU generation, all steps were conducted with our own Perl scripts. BLAST analyses were performed on all reads against the NCBI nucleotide database, and the results were further processed for taxonomic assignations using MEGAN. An optional score adjustment was used when paired reads matched the same species. The required parameters of the LCA assignment were set as minimum support $=2$, minimum score $=80$, top percentage $=1$ (Murray et al. 2011; Montaña et al. 2012). Classification results were manually checked to correct the ambiguous assignation caused by synonyms for fungal species or an ambiguous annotation in the NCBI database.

Evaluating biodiversity based on metagenomic data

As recommended by Haegeman et al. (2013), Shannon's and Gini-Simpson's diversity indices (Shannon 1948; Rao 1982) were adopted to estimate the alpha diversity in the fungal community. Shannon's index is affected by the species number and their equitability, or evenness. A greater number of species and an even distribution of abundances result in an elevated Shannon's diversity index. The maximum Shannon's diversity index for a sample indicates that all species are nearly equally abundant. The Gini-Simpson's diversity index is measured as the probability that two individuals randomly selected from a sample belong to the same species, with a range from 0 to 1 . Value of 0 indicates lack of diversity, i.e., one dominant species or taxon in the community, and 1 suggests that the community contains an infinite number of taxa with all taxa present equally. Before alpha-diversity indices were calculated, multiple rarefactions were performed with our own Perl scripts. All fungal reads from each marker were resampled starting at the depth of 1,000 reads, stepping up to 385,000 reads with increments of 1,000 , and ten replicates were done at each sampling depth. For illustrating fungal diversities, taxonomic relationships of all detected fungal genera were converted to the Newick format and uploaded to the web-based tool Interactive Tree Of Life v2.2 (Letunic and Bork 2011), and the taxonomic trees for each barcode and for all barcodes combined were generated.

Estimation of the taxon abundance based on copy numbers of PCR-amplified DNA reads for a mixture of homologous genes in a multi-template PCR can be biased due to the differences in the primer binding energy to the target (Kanagawa 2003). Consequently, the taxon diversity and proportion of any given operational taxonomic unit (OTU) in the fungal community are expected to differ when using different sets of DNA barcodes. In this study, the percentage of reads for a taxon was calculated by dividing the total reads of fungi generated by individual barcodes (Table S3). Because of the bias in the taxonomic assignations of mtATP6, that was restricted to the class Agaricomycetes except for six reads, we 
excluded mtATP6 from estimating species abundance with multiple barcodes. The percentage of reads for each of the genera generated from five barcodes (ITS1/2, ITS3/4, nrLSULR, nrLSU-U and mtLSU) was then transformed to a rank score based on the abundance of each genus in the community using the formula $20-19($ rank -1$) /(N-1)$. The ranks $(1,2$, 3 ...to $N$ ) represent the order of abundance (percentage of reads) for all taxa; thus, a taxon with rank 1 is most abundant and receives the highest rank score (20). When several taxa have the same abundance, the highest rank of these taxa was used as representative. The highest rank score was set to 20 for a given taxon having the highest number of reads (rank $=1)$, and the lowest rank score was set to 1 for a given taxon having lowest number of reads $($ rank $=N)$.

\section{Results}

Phylogenetic diversity of fungal community based on PCR cloning and Sanger sequencing of ITS1/ITS4

NrITS was amplified using the ITS1/ITS4 barcode for analyzing the composition of the endophytic fungal community in the orchid roots. Of the 500 nrITS sequences obtained and analyzed, a BLAST search assigned $76.4 \%$ of the sequences to fungi, of which only 19 genera (29 taxa) were identified (Table 1). The top 10 most abundant fungal taxa were Penicillium sp. (20.0\%), Trechispora farinacea (17.2\%), Leotiomyceta (12.0\%), Exophiala (6.6\%), Fusarium solani (4.4\%), Cladosporium sp. (3.6\%), Epulorhiza sp. Van44 (2.4\%), Alternaria sp. (2.0\%), Leucocoprinus birnbaumii $(2.0 \%)$, and Sporothrix inflata $(1.2 \%)$.

Efficiency of six barcoding markers in fungal identification by metagenomics

In total, 27,099,433 PE reads were obtained and sorted according to the six markers from the raw sequencing data. After single-copy haplotypes were removed, 21,009,068 (77.5\%) $\mathrm{PE}$ reads remained and were further clustered into OTUs. Among these markers, nrLSU-U yielded the most reads assigned to fungi $(90.7 \%$ of $6,636,430)$, followed by mtLSU $(69.7 \%$ of $8,132,397)$, mtATP6 $(99.3 \%$ of $2,187,555)$, ITS1/2 $(86.1 \%$ of $1,504,231)$, ITS3/4 $(79.1 \%$ of 649,608$)$, and nrLSU-LR $(20.3 \%$ of $1,898,847)$. No correlation existed between the read numbers and the number of assigned fungal OTUs. The coverage (number of reads/number of OTUs) of markers ranged from $1,338 \times$ of nrLSU-LR to $36,191 \times$ of mtATP6. Taxon assignation using a MEGAN analysis showed that $32.8-59.5 \%$ of OTUs could be assigned to fungi, except for mtATP6 (12.0\%) (Table 2). In contrast, $40.6 \%$ of OTUs amplified with ITS1/ITS2, $22.7 \%$ with ITS3/ITS4, $5.7 \%$ with nrLSU-LR, $26.6 \%$ with nrLSU-U, $34.4 \%$ with mtLSU and $83.8 \%$ with mtATP6 were not assignable to any organisms based on the BLAST searches (Table 2). Although most reads for mtATP6 were assigned to fungi, $96.2 \%$ of these reads belonged to one OTU (Ceratobasidium sp. CBS 189.90).

Fungal diversity in orchid roots detected with six barcoding markers

Six phyla (Ascomycota, Basidiomycota, Chytridiomycota, Entomophthoromycota, Glomeromycota, Neocallimastigomycota) and three subphyla (Kickxellomycotina, Mucoromycotina, Motierellomycotina) were detected in Phalaenopsis roots (Tables 3, S2). Both major phyla, Ascomycota and Basidiomycota, were detected by all markers, while the remaining phyla/subphyla were only detected with the markers for the nrITS and nrLSU regions, revealing insufficiencies of mitochondrial markers. Glomeromycota, Neocallimastigomycota, and Kickxellomycotina were only observed with single markers, whereas Chytridiomycota, Entomophthoromycota, Mortierellomycotina, and Mucoromycotina were detected with two or more markers. As indicated, Ascomycota and Basidiomycota were dominant. All nrITS markers yielded a higher abundance of Ascomycota, while nrLSU and mitochondrial markers yielded a higher abundance of Basidiomycota (Fig. 1a). At the class level, the dominant classes were Dothideomycetes (Ascomycota), Eurotiomycetes (Ascomycota), Sordariomycetes (Ascomycota), and Agaricomycetes (Basidiomycota), which nevertheless displayed high variances in the relative abundance across markers (Fig. 1b). For example, the detection of Dothideomycetes was mostly restricted to ITS1/2, low abundance of the Sordariomycetes was observed when using nrLSU-LR, and the abundance of Agaricomycetes ranged from 20 to $94 \%$ across five markers. At the order level, 34 orders were identified with markers of ITS1/2, 31 for nrLSULR, 35 for ITS3/4, 46 for nrLSU-U, 19 for mtLSU, and 6 orders for mtATP6. At the generic level, 76 genera were detected with markers for ITS1/2, 38 for ITS3/4, 33 for nrLSU-LR, 111 for nrLSU-U, 33 for mtLSU, and 8 for mtATP6 (Table 3). With all markers integrated, 10 phyla/ subphyla, 19 classes, 64 orders, and 205 genera were detected in this study (Fig. 2, Table 3).

Multiple rarefactions and alpha-diversity estimations

As the total numbers of sequences varied across the six markers, from the lowest of 385,278 with ITS3/4 to the highest of 6,018,234 with nrLSU-LR, multiple rarefactions were performed on markers to minimize the bias resulting from unequal sequencing depths. ITS $1 / 2$, ITS $3 / 4$, and nrLSU-U showed similar resolutions at low sequencing 
Table 1 Taxonomic assignations and counts of endophytic species in Phalaenopsis KC1111 identified by gene cloning and Sanger sequencing of ITS $1 / 4$ regions

\begin{tabular}{|c|c|c|c|c|c|}
\hline Phylum & Class & Order & Genus & Taxonomic assignation & Counts \\
\hline \multirow[t]{17}{*}{ Ascomycota } & & & & Leotiomyceta & 60 \\
\hline & & & & Ascomycota & 2 \\
\hline & Dothideomycetes & Capnodiales & Cladosporium & Cladosporium & 18 \\
\hline & & & Devriesia & Devriesia strelitziicola & 1 \\
\hline & & Pleosporales & Thyridaria & Thyridaria & 1 \\
\hline & & & Alternaria & Alternaria & 10 \\
\hline & Eurotiomycetes & & & Eurotiomycetes & 3 \\
\hline & & Chaetothyriales & Cladophialophora & Cladophialophora bantiana & 1 \\
\hline & & & Exophiala & Exophiala & 32 \\
\hline & & & & Exophiala moniliae & 1 \\
\hline & & Eurotiales & Penicillium & Penicillium & 100 \\
\hline & Saccharomycetes & Saccharomycetales & & Saccharomycetales & 2 \\
\hline & Sordariomycetes & Hypocreales & Sarocladium & Sarocladium strictum & 1 \\
\hline & & & Trichoderma & Trichoderma & 2 \\
\hline & & & Fusarium & Fusarium solani & 22 \\
\hline & & & & Fusarium & 2 \\
\hline & & Ophiostomatales & Sporothrix & Sporothrix inflata & 6 \\
\hline \multirow[t]{11}{*}{ Basidiomycota } & & Erythrobasidiales & Occultifur & Occultifur aff. externus IMUFRJ 52019 & 1 \\
\hline & & & & Occultifur externus & 1 \\
\hline & & & Rhodotorula & Rhodotorula calyptogenae & 1 \\
\hline & & Sporidiobolales & & Sporidiobolales & 1 \\
\hline & Agaricomycetes & Agaricales & Leucocoprinus & Leucocoprinus Birnbaumii & 10 \\
\hline & & Cantharellales & Epulorhiza & Epulorhiza sp. Van44 & 12 \\
\hline & & Polyporales & Nigroporus & Nigroporus vinosus & 1 \\
\hline & & Trechisporales & Trechispora & Trechispora farinacea & 86 \\
\hline & & & & Trechispora & 2 \\
\hline & Agaricostilbomycetes & & Rhodotorula & Rhodotorula bloemfonteinensis & 2 \\
\hline & Tremellomycetes & Tremellales & Cryptococcus & Cryptococcus podzolicus & 1 \\
\hline \multirow[t]{5}{*}{ Other organisms } & & & & Alveolata & 5 \\
\hline & & & & Bacteria & 1 \\
\hline & & & & Eukaryota & 6 \\
\hline & & & & Metazoa & 5 \\
\hline & & & & Viridiplantae & 40 \\
\hline Not assigned & & & & & 61 \\
\hline Total & & & & & 500 \\
\hline
\end{tabular}

depths, as indicated by the curves of these markers that overlapped when the rarefied number was less than 100,000 (Fig. 3). Nevertheless, as the number of sequences increased, nrLSU-U demonstrated the best resolution (442.4 OTUs of 385,000 sequences) compared with other markers, followed by ITS1/2 (371.4 OTUs) and ITS3/4 (333.8 OTUs). We further estimated the alpha diversity of the fungal community with the rarefied data set. The two alpha diversity indicators, Shannon's and Gini-Simpson's indices, were adopted due to their stability and robustness in metagenomic analyses (Haegeman et al. 2013). Table 4 shows the rarefied Shannon's and Gini-Simpson's indices for floras uncovered by markers, in which ITS1/2 (2.49 and 0.85 for Shannon's and GiniSimpson's indices, respectively) displayed higher specie richness than ITS3/4 (2.02 and 0.78), nrLSU-U (1.83 and 0.76), nrLSU-LR (1.47 and 0.68), mtLSU (1.09 and 0.58), and mtATP6 (0.18 and 0.07). Both indices showed that the nrITS regions had better resolution in width and depth in uncovering 
Table 2 Summary of sequencing reads and operational taxonomic unit (OTU) numbers from all barcodes

\begin{tabular}{lcccccc}
\hline & ITS1/2 & ITS3/4 & nrLSU-LR & nrLSU-U & mtLSU & mtATP6 \\
\hline Reads & & & & & & \\
Total & $2,050,657$ & 948,313 & $2,854,004$ & $9,249,520$ & $9,454,223$ & $2,542,716$ \\
Processed to OTU & $1,504,231$ & 649,608 & $1,898,847$ & $6,636,430$ & $8,132,397$ & $2,187,555$ \\
Fungi & $1,294,385$ & 513,844 & 385,244 & $6,018,234$ & $5,670,611$ & $2,171,475$ \\
Not assigned & 149,192 & 26,313 & 2,735 & 551,261 & 746,746 & 15,482 \\
Other kingdoms & 60,654 & 109,451 & $1,510,868$ & 66,935 & $1,715,040$ & 598 \\
OTU & & & & & 1,176 & \\
Total & 1,177 & 746 & 878 & & \\
Fungi & $512(43.5 \%)$ & $364(48.8 \%)$ & $287(32.7 \%)$ & $1,189(59.5 \%)$ & $387(32.9 \%)$ & $60(12.0 \%)$ \\
Not assigned & $478(40.6 \%)$ & $169(22.7 \%)$ & $50(5.7 \%)$ & $532(26.6 \%)$ & $404(34.4 \%)$ & $420(83.8 \%)$ \\
Other kingdoms & $187(15.9 \%)$ & $213(28.6 \%)$ & $541(61.6 \%)$ & $276(13.8 \%)$ & $385(32.7 \%)$ & $21(4.2 \%)$ \\
\hline
\end{tabular}

the biodiversity than nrLSU and mitochondrial regions (Table 4).

Data analysis using rank scoring to evaluate fungal diversity

The taxonomic assignment for the ten most abundant OTUs for each marker is shown in Table S4. Unexpectedly, different dominant species were identified among markers. The most abundant OTUs were assigned as Alternaria, Penicillium, Trechispora, Trechispora, Serpula, and Ceratobasidium detected with ITS1/2, ITS3/4, nrLSU-LR, nrLSU-U, mtLSU and
mtATP6, respectively. As each marker only represented a part of the fungal community, the data across these markers must be combined to get an overview of the microbiome. Here, a rank-scoring strategy was developed for integrating the information on species composition obtained from multiple markers. Value 0 suggests no reads detected. Abundance of each genus in the community was calculated by summing the rank scores for the five barcodes used; results for mtATP6 were excluded due to its biased detection toward Agaricomycetes. In the rank-scoring, the top 15 genera were Penicillium (including teleomorph Talaromyces), Sporothrix
Table 3 Summary of taxonomic assignations and species diversity using six markers

\begin{tabular}{|c|c|c|c|c|c|c|}
\hline Assignation & ITS $1 / 2$ & ITS3/4 & nrLSU-LR & nrLSU-U & $\mathrm{mtLSU}$ & mtATP6 \\
\hline Fungal reads & $1,294,385$ & 513,844 & 385,278 & $6,018,234$ & $5,670,611$ & $2,171,475$ \\
\hline Assigned to phylum level & $1,285,639$ & 504,494 & 322,245 & $6,012,781$ & $5,867,195$ & $2,171,471$ \\
\hline Assigned to order level & 967,973 & 130,424 & 319,267 & $4,267,361$ & $5,618,342$ & $2,170,485$ \\
\hline Assigned to genus level & 871,208 & 73,730 & 283,860 & $4,025,934$ & $5,616,600$ & $2,170,410$ \\
\hline Fungal OTUs & 512 & 364 & 288 & 1,189 & 387 & 60 \\
\hline Assigned to phylum level & 492 & 345 & 252 & 1,163 & 376 & 58 \\
\hline Assigned to class level & 405 & 248 & 208 & 943 & 339 & 57 \\
\hline Assigned to order level & 381 & 224 & 159 & 822 & 319 & 50 \\
\hline Assigned to genus level & 260 & 132 & 112 & 487 & 260 & 43 \\
\hline \multicolumn{7}{|l|}{ Phylum/subphylum } \\
\hline Ascomycota & 354 & 257 & 123 & 883 & 328 & 2 \\
\hline Basidiomycota & 130 & 74 & 117 & 267 & 48 & 56 \\
\hline Chytridiomycota & & 2 & 4 & 2 & & \\
\hline Entomophthoromycota & & 2 & 2 & & & \\
\hline Glomeromycota & & 2 & & & & \\
\hline Neocallimastigomycota & & & 1 & & & \\
\hline Kickxellomycotina & & 1 & & & & \\
\hline Mortierellomycotina & 7 & 3 & 3 & 6 & & \\
\hline Mucoromycotina & 1 & 4 & 2 & 5 & & \\
\hline Identified orders (Total 64) & 34 & 31 & 35 & 46 & 19 & 6 \\
\hline Identified genera (Total 201) & 76 & 38 & 32 & 111 & 33 & 8 \\
\hline
\end{tabular}


Fig. 1 Read distribution of sequences according to phylum (a) and class (b) of fungi in roots of greenhouse-grown Phalaenopsis KC1111. Bar colors denote the taxon detected by each marker a

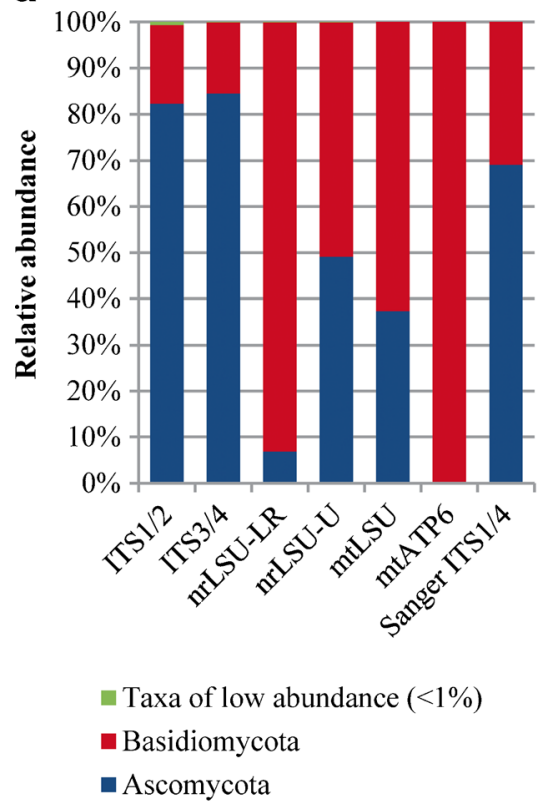

b

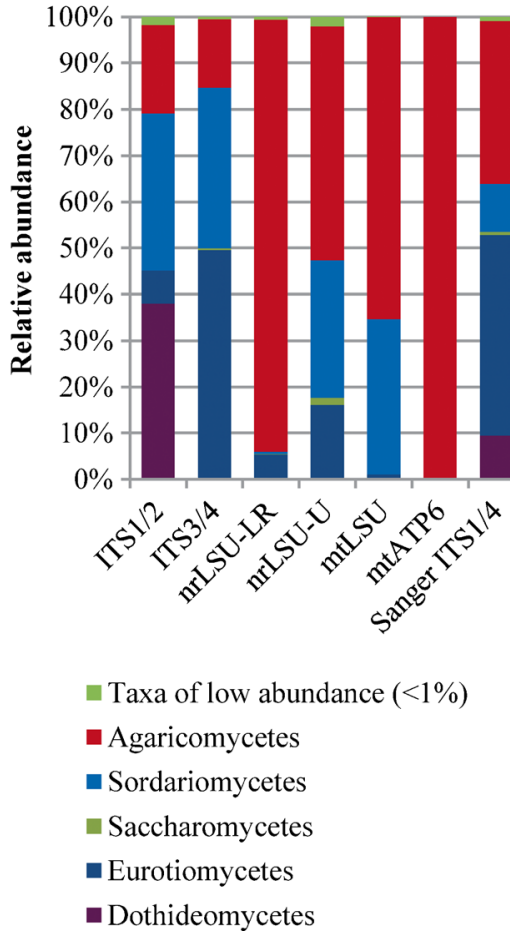

(including teleomorph Ophiostoma), Trechispora, Fusarium (including teleomorph Gibberella), Candida, Cladosporium, Mortierella, Exophiala, Meira, Aspergillus, Devriesia, Leucocoprinus, Mycospharella, Trichoderma (including teleomorph Hypocrea), and Cladophialophora, all having rank scores between 40.34 and 84.21 (Fig. 4, Table S5).

\section{Discussion}

DNA barcoding for species identification

Although molecular techniques using cloning and Sanger sequencing largely avoid the difficulties of microbial culture or morphotype identification, in the present study, sequencing the ITS1/4 region to investigate the fungal species diversity in orchid roots only identified 29 taxa from 500 clones. Even so, of the top 10 abundant genera (Table 1), nine were also recognized as the dominant genera in the metagenomic analyses (Table S5): Penicillium (20.0\%; meta-rank 2 in the NGS approach), Trechispora (17.6\%; meta-rank 3), Exophiala (6.6 \%; meta-rank 8), Fusarium (4.8\%; meta-rank 4), Cladosporium (3.6\%; meta-rank 6), Alternaria (2.0\%; meta-rank 17), Leucocoprinus (2.0\%; meta-rank 12), Sporothrix (1.2\%; meta-rank 1), and Trichoderma $(0.4 \%$; meta-rank 14). High repeatability in both methods reflects that Sanger sequencing may be capable of detecting common taxa. However, this conventional molecular tool lacks breadth in screening the microbial community, as indicated by the detection of a wide range of classes/orders of fungi through the metagenomic analyses with NGS; for the constituent species, 462 ITS1/2-OTUs (90.2\%) and 342 ITS3/4-OTUs $(94.0 \%)$ were minor with frequencies lower than $0.2 \%$, a frequency equivalent to 1 detection from 500 clones, reflecting the power of deep sequencing (Mardis 2008).

Primer preference undoubtedly biases estimations of the species composition in a community (Bellemain et al. 2010). In this study, up to one third of the OTUs detected using the mtLSU were assigned to bacteria, likely from the low specificity of the primers for fungi (Table 2). The mtLSU primers were designed for conserved regions of the large subunit rDNA of the mitochondrion, which share high similarities with bacterial ribosomal components (Kanagawa 2003). Likewise, the low efficiency of the nrLSU-LR barcode in detecting fungal species may also have resulted from low primer specificity, as shown by the fact that $\sim 80 \%$ of the reads were assigned to plants instead of fungi. Even so, the nrLSU-LR was useful for identifying 17 unique genera (Table S4). Another extreme was with the mtATP6 amplification, that yielded all of the reads belonging to the Basidiomycota, $95.5 \%$ of which were assigned to Ceratobasidium, a mycorrhizal genus associated with orchids (Irwin et al. 2007). On the other hand, $83.8 \%$ of the mtATP6 OTUs representing $0.7 \%$ of the reads remained unidentified likely due to insufficient information of mtATP6 sequences. All of these facts revealed high inconsistency across barcodes. Apparently, using one or few barcodes likely increases the risks of misidentifying the 


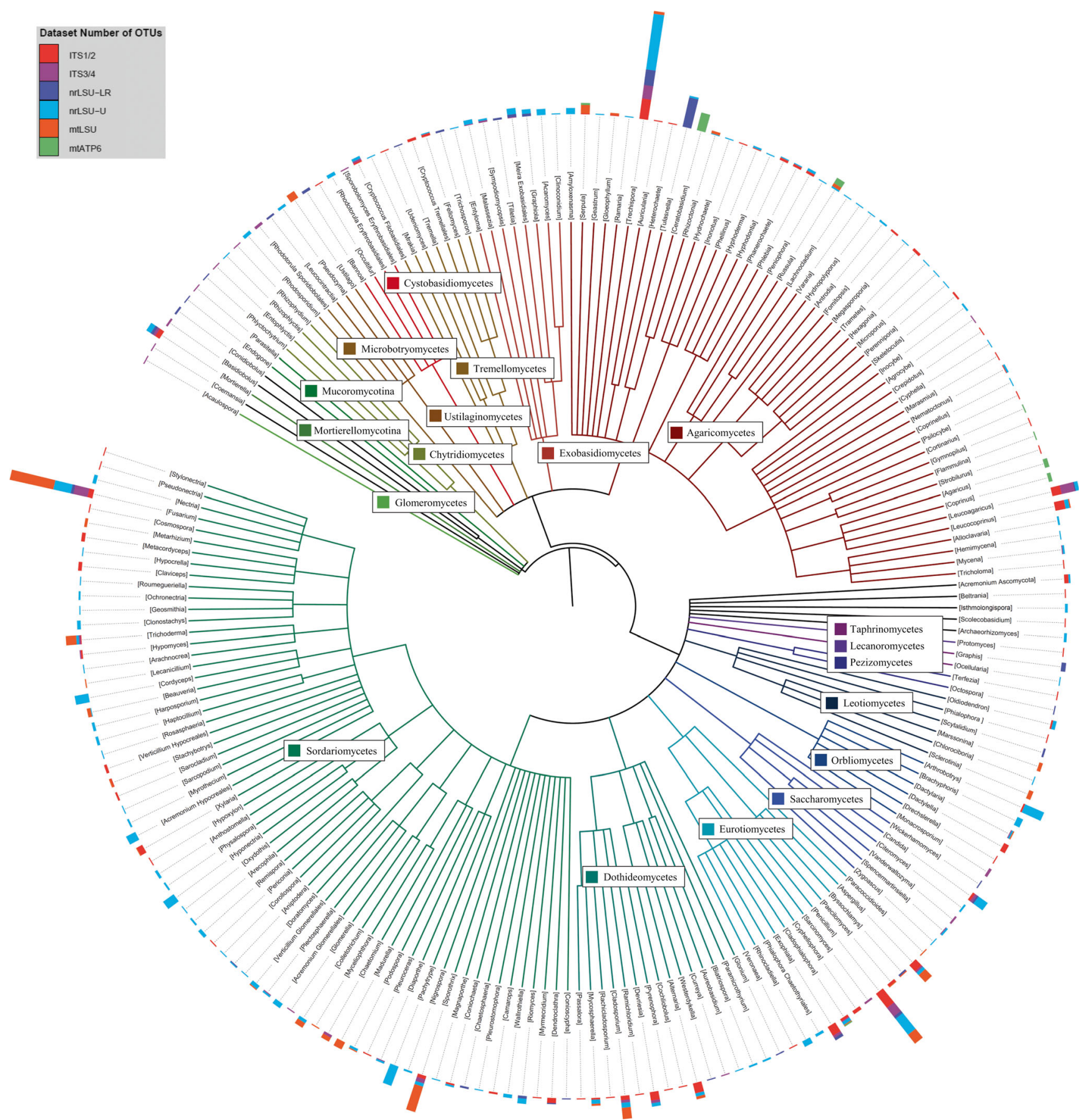

Fig. 2 Hierarchical tree representing taxonomic relationships of fungal genera detected in roots of greenhouse-grown Phalaenopsis. Branch colors indicate the classes (in boxes) of the OTUs. The height of the bars

species composition in a microbial community, although nrITS is one of the best barcodes for fungal species discrimination (Schoch et al. 2012). Using multiple barcodes is therefore necessary and has been strongly recommended (Nilsson et al. 2008; Gazis et al. 2011). Among the barcodes utilized in this study, ITS1/2, ITS3/4, and nrLSU-U were the most competent in uncovering the diversity of the fungal community in Phalaenopsis roots (Fig. 1), while mitochondrial markers in the circle outside the branch tips corresponds to the number of OTUs within genera. The key to bar color for the markers is at the top right

(mtLSU and mtATP6) yielded a low alpha diversity with rarely detected genera (Tables 3 and 4).

Species composition and ecological roles of constituent fungi within orchid roots

Orchid roots represent an ecosystem that fosters a high diversity of microbial species. Noticeably, genetic barcodes 


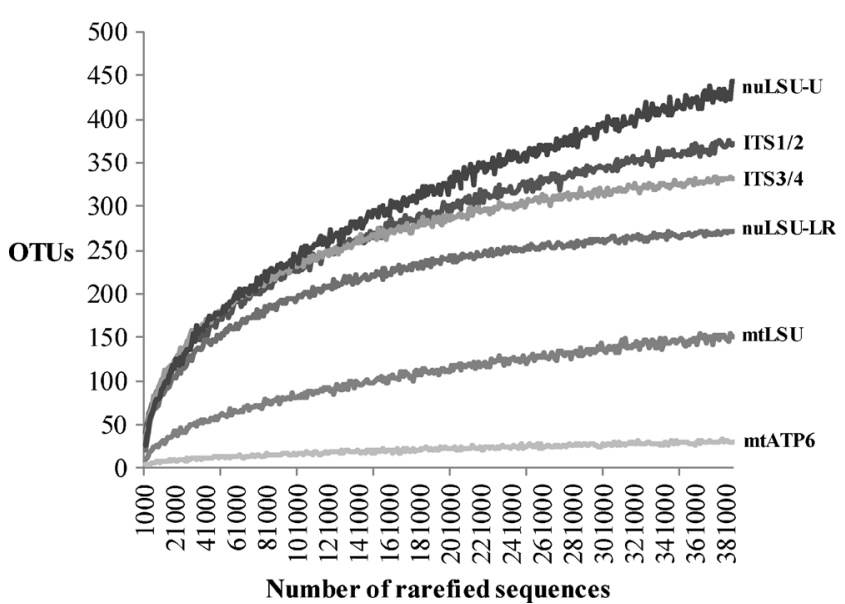

Fig. 3 OTU accumulation curves of multiple rarefactions with six markers sequenced with Illumina GAIIx

identified different floristic compositions at the class level (Fig. 2) and different common species from the same root community (Table S4). For example, for various barcodes, the most common species (with percentage reads) were as follows: ITS1/2, Alternaria sp. (up to $30.4 \%$ ); ITS3/4, Penicillium sp. (37.8 \%); nrLSU-LR, Trechispora farinacea (48.9 \%); nrLSU-U, Trechispora sp. (39.2\%); mtLSU, Serpula sp. (64.7\%). Such inconsistencies across barcodes create doubts that genetic markers can accurately uncover the species composition of an ecological community. Our data show that different barcode primers tend to have different annealing kinetics to the target DNA in PCR with multitemplate samples. In addition, fungal DNA sequence information for barcoding in GenBank is incomplete, thus lowering the power to identify species (Schloss et al. 2011; Pinto and Raskin 2012). Nonetheless, taxa frequently identified across barcodes were likely to represent dominant elements in the fungal community.

Since taxa were preferentially detected across different barcodes, the species richness cannot be simply estimated by averaging the read percentages of taxa (e.g., genus) from each barcode. For example, as high as $65 \%$ of the reads amplified with mtLSU were assigned to Serpula, which would account for the second-most abundant genus by average (13.0\%) across five barcodes, whereas Fusarium, Penicillium, and Sporothrix, detected with five barcodes, turned out to be minor constituents, having average read percentages of $9.0 \%, 8.0 \%$, and $3.3 \%$, respectively (Table S3). By assigning the OTUs into ranks based on the relative abundance (Table S5) using our rank-scoring, we could minimize the calculation bias encountered with data combination. With this new approach, multiple barcodes are easy to integrate for estimating species richness.

Nine of the ten most abundant genera have been reported as fungi that promote the growth of plants, including Trechispora (meta-rank 3) and Mortierella (meta-rank 7), that are likely involved in mycorrhizal formation (Ochora et al. 2001; Rinaldi et al. 2008) (Fig. 4, Table S2). Although Dearnaley et al. (2012) did not report any ecological functions for these fungi, they are potentially useful for horticulture. Given that the nature of the interactions between these fungi and orchids is uncertain, the role of Trechispora farinacea, a dominant species in the root community (Table S4), needs to be further examined using inoculation experiments. Cultural conditions should be optimized specifically for these symbiotic fungi.

Of the 21 other mycorrhizal genera identified in the present study (Tables 1, S2), Tulasnella (anamorphic Epulorhiza) and Ceratobasidium are common symbionts with orchids (Suárez et al. 2006; Irwin et al. 2007; Otero et al. 2007; Dearnaley et al. 2012; Graham and Dearnaley 2012). Tulasnella is involved in the symbiotic germination of Chiloglottis aff. jeanesii and C. valida (Roche et al. 2010), whereas an isolate of Ceratobasidium is potentially useful for the biocontrol of Erwinia chrysanthemi, the bacterium causing soft rot in Phalaenopsis (Wu et al. 2011). Thus, Tulasnella and Ceratobasidium spp. are likely to be important mycorrhizal species coexisting with Phalaenopsis. Although more than $80 \%$ of the land plant families are thought to have symbiotic relationships with arbuscular mycorrhizal fungi (AMF, Glomeromycota) (Kawaguchi and Minamisawa 2010), Acaulospora was the only AMF genus that we detected. Failing to detect other AMF may be ascribed to the short read length with Illumina sequencing (cf. Stockinger et al. 2010).

Moreover, Penicillium species (meta-rank 1 here) are common endophytes in plants (Vega et al. 2006), and some species can improve phosphate solubility or produce gibberellic acid to stimulate plant growth (Wakelin et al. 2007; Khan et al. 2008). Fungi may also function as biocontrol agents (e.g., Meira and Candida; Nguyen et al. 2011) or nematode predators (e.g., Dactyllela and Arthrobotrys; Schenck et al. 1977). Nematodes, common invertebrates in orchids, often cause leaf yellowing and reduce plant vigor (Kuehnle 2006). Such nematophagous fungi may thus play a critical role in controlling nematode infection in orchids. Using symbiotic fungi for controlling disease outbreak or improving the resistance to pathogens has been demonstrated for orchids and crops (cf. Lee et al. 2009; Wu et al. 2011; Mosquera-Espinosa et al.
Table 4 Indices of alpha diversity across markers

\begin{tabular}{lllllll}
\hline Diversity index & ITS1/2 & ITS3/4 & nrLSU-LR & nrLSU-U & mtLSU & mtATP6 \\
\hline Shannon & 2.49 & 2.02 & 1.47 & 1.83 & 1.09 & 0.18 \\
Gini-Simpson & 0.85 & 0.78 & 0.68 & 0.76 & 0.55 & 0.07 \\
\hline
\end{tabular}


Fig. 4 Bar plot of rank scores at the genus level. Rank scores obtained from five markers are represented in different grayscale colors

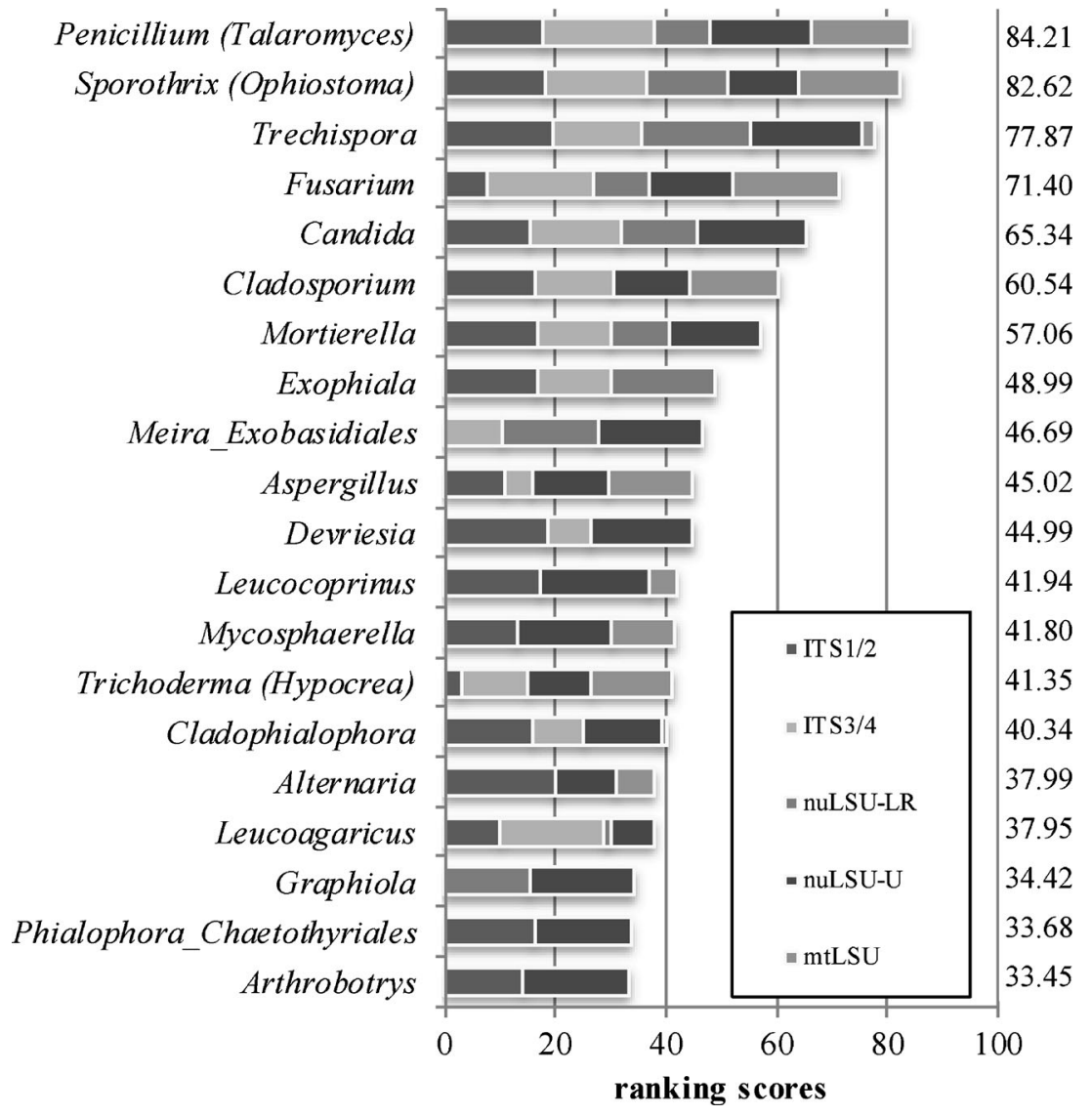

2013). Another benefit might be conferred by Sporothrix (meta-rank 2); its abundance is likely associated with the good growth of orchids in Sphagnum moss, a popular potting material in the orchid industry in which Sporothrix is frequently found (Zhang and Andrews 1993; Feeney et al. 2007).

Among the well-documented, common pathogenic fungi that infect orchids, Fusarium (meta-rank 4) and Colletotrichum (meta-rank 26) were also detected in this study. Symptoms may be severe enough to impair the growth of Phalaenopsis, e.g., some Fusarium species lead to wilting of orchids (Benyon et al. 1996; Divakaran et al. 2008), and Colletotrichum species cause anthracnose disease (Yang et al. 2011). However, pathogenic species do not always trigger necrotic symptoms because of a lag in symptom expression early during infection (Newton et al. 2010) or the presence of antagonistic species that repress pathogenicity (Schulz and Boyle 2005).

\section{Conclusions}

Metagenomic analysis with NGS techniques provides not only a vast amount of data of barcode sequences, but deep insights into the species composition of a fungal community. Here, multiple barcodes were used to resolve the taxa within a microbial community; 152 genera ( $73.8 \%$ OTUs) appeared only in the barcoding with single markers, indicating that no single barcode was able to disclose the diverse microflora comprehensively. Of the six barcodes, ITS1/2, ITS3/4, and nrLSU-U worked the best to decipher the microbiome in Phalaenopsis roots. Our metagenomic analyses suggested that species of the mycorrhizal Trechispora and Mortierella might play some key roles in promoting orchid vigor. Methodological approaches, e.g., in silico simulations on primer preferences, deciphering mock communities with multiple markers, and isolating potentially useful fungi for whole genome sequencing, can be conducted in the future. tional Cheng Kung University and the National Science Council, Taiwan.

Open Access This article is distributed under the terms of the Creative Commons Attribution License which permits any use, distribution, and reproduction in any medium, provided the original author(s) and the source are credited.

\section{References}

Alef K, Nannipieri P (1995) Methods in applied soil microbiology and biochemistry. Academic, London
Acknowledgments This study was financially supported by the Na- 
Arditti J (1992) Fundamentals of orchid biology. Wiley, New York

Beckman CH (1987) The nature of wilt diseases of plants. APS Press, California

Bellemain E, Carlsen T, Brochmann C, Coissac E, Taberlet P, Kauserud H (2010) ITS as an environmental DNA barcode for fungi: an in silico approach reveals potential PCR biases. BMC Microbiol 10:189

Benyon F, Summerell B, Burgess L (1996) Association of Fusarium species with root rot of Cymbidium orchids. Australas Plant Pathol 25:226-228

Berendsen RL, Pieterse CMJ, Bakker PAHM (2012) The rhizosphere microbiome and plant health. Trends Plant Sci 17:478-486

Bisseling T, Dangl JL, Schulze-Lefert P (2009) Next-generation communication. Science 324:691

Burgeff H (1959) Mycorrhiza of orchids. In: Withner C (ed) The orchids. Ronald, New York, pp 361-395

Cating R, Palmateer A, McMillan R Jr (2009) First report of Sclerotium rolfsii on Ascocentrum and Ascocenda orchids in Florida. Plant Dis 93:963

Cowan D, Meyer Q, Stafford W, Muyanga S, Cameron R, Wittwer P (2005) Metagenomic gene discovery: past, present and future. Trends Biotechnol 23:321-329

Dearnaley J, Martos F, Selosse M-A (2012) Orchid mycorrhizas: molecular ecology, physiology, evolution and conservation aspects. In: Hock B (ed) Fungal associations. Springer, Berlin, pp 207-230

DeSalle R, Graham SW, Fazekas AJ, Burgess KS, Kesanakurti PR, Newmaster SG, Husband BC, Percy DM, Hajibabaei M, Barrett SCH (2008) Multiple multilocus DNA barcodes from the plastid genome discriminate plant species equally well. PLoS ONE 3:e2802

Divakaran M, Geetha S, Nirmal Babu K, Peter K (2008) Isolation and fusion of protoplasts in Vanilla species. Curr Sci 94:115-120

Doyle J, Doyle J (1987) Genomic plant DNA preparation from fresh tissue-CTAB method. Phytochem Bull 19:11-15

Druzhinina IS, Kopchinskiy AG, Komoń M, Bissett J, Szakacs G, Kubicek CP (2005) An oligonucleotide barcode for species identification in Trichoderma and Hypocrea. Fungal Genet Biol 42:813-828

Feeney KT, Arthur IH, Whittle AJ, Altman SA, Speers DJ (2007) Outbreak of sporotrichosis, Western Australia. Emerg Infect Dis 13:1228

Gazis R, Rehner S, Chaverri P (2011) Species delimitation in fungal endophyte diversity studies and its implications in ecological and biogeographic inferences. Mol Ecol 20:3001-3013

Graham RR, Dearnaley JDW (2012) The rare Australian epiphytic orchid Sarcochilus weinthalii associates with a single species of Ceratobasidium. Fungal Divers 54:31-37

Grubisha LC, Levsen N, Olson MS, Lee Taylor D (2012) Intercontinental divergence in the Populus-associated ectomycorrhizal fungus, Tricholoma populinum. New Phytol 194:548-560

Haegeman B, Hamelin J, Moriarty J, Neal P, Dushoff J, Weitz JS (2013) Robust estimation of microbial diversity in theory and in practice. ISME J 7:1092-1101

Hebert PD, Gregory TR (2005) The promise of DNA barcoding for taxonomy. Syst Biol 54:852-859

Hinsinger P, Bengough AG, Vetterlein D, Young IM (2009) Rhizosphere: biophysics, biogeochemistry and ecological relevance. Plant Soil 321:117-152

Hirsch PR, Mauchline TH, Clark IM (2010) Culture-independent molecular techniques for soil microbial ecology. Soil Biol Biochem 42: 878-887

Huson DH, Mitra S, Ruscheweyh H-J, Weber N, Schuster SC (2011) Integrative analysis of environmental sequences using MEGAN4. Genome Res 21:1552-1560

Irwin MJ, Bougoure JJ, Dearnaley JDW (2007) Pterostylis nutans (Orchidaceae) has a specific association with two Ceratobasidium root-associated fungi across its range in eastern Australia. Mycoscience 48:231-239
Kaeberlein T (2002) Isolating "uncultivable" microorganisms in pure culture in a simulated natural environment. Science 296:1127-1129

Kanagawa T (2003) Bias and artifacts in multitemplate polymerase chain reactions (PCR). J Biosci Bioeng 96:317-323

Kawaguchi M, Minamisawa K (2010) Plant-microbe communications for symbiosis. Plant Cell Physiol 51:1377-1380

Khan S, Hamayun M, Yoon H, Kim H-Y, Suh S-J, Hwang S-K, Kim J-M, Lee I-J, Choo Y-S, Yoon U-H, Kong W-S, Lee B-M, Kim J-G (2008) Plant growth promotion and Penicillium citrinum. BMC Microbiol 8:231

Kõljalg U, Larsson KH, Abarenkov K, Nilsson RH, Alexander IJ, Eberhardt U, Erland S, Høiland K, Kjøller R, Larsson E (2005) UNITE: a database providing web-based methods for the molecular identification of ectomycorrhizal fungi. New Phytol 166:1063-1068

Konow EA, Wang Y-T (2001) Irradiance levels affect in vitro and greenhouse growth, flowering, and photosynthetic behavior of a hybrid Phalaenopsis orchid. J Am Soc Hortic Sci 126:531-536

Kuehnle AR (2006) Orchids. In: Anderson NO (ed) Flower breeding and genetics. Springer, Dordrecht, pp 539-560

Lee SO, Kim HY, Choi GJ, Lee HB, Jang KS, Choi YH, Kim JC (2009) Mycofumigation withOxyporus latemarginatusEF069 for control of postharvest apple decay and Rhizoctonia root rot on moth orchid. J Appl Microbiol 106:1213-1219

Letunic I, Bork P (2011) Interactive tree of life v2: online annotation and display of phylogenetic trees made easy. Nucleic Acids Res 39: W475-W478

Mardis ER (2008) The impact of next-generation sequencing technology on genetics. Trends Genet 24:133-141

Mendes R, Kruijt M, de Bruijn I, Dekkers E, van der Voort M, Schneider JH, Piceno YM, DeSantis TZ, Andersen GL, Bakker PA (2011) Deciphering the rhizosphere microbiome for disease-suppressive bacteria. Science 332:1097-1100

Montaña JS, Jiménez DJ, Hernández M, Ángel T, Baena S (2012) Taxonomic and functional assignment of cloned sequences from high Andean forest soil metagenome. Antonie Van Leeuwenhoek 101:205-215

Mosquera-Espinosa AT, Bayman P, Prado GA, Gómez-Carabalí A, Otero JT (2013) The double life of Ceratobasidium: orchid mycorrhizal fungi and their potential for biocontrol of Rhizoctonia solani sheath blight of rice. Mycologia 105:141-150

Murray DC, Bunce M, Cannell BL, Oliver R, Houston J, White NE, Barrero RA, Bellgard MI, Haile J (2011) DNA-based faecal dietary analysis: a comparison of qPCR and high throughput sequencing approaches. PLoS ONE 6:e25776

Newton AC, Fitt BDL, Atkins SD, Walters DR, Daniell TJ (2010) Pathogenesis, parasitism and mutualism in the trophic space of microbe-plant interactions. Trends Microbiol 18:365-373

Nguyen MT, Ranamukhaarachchi DB, Senaratne L (2011) Efficacy of antagonist strains of Bacillus megaterium, Enterobacter cloacae, Pichia guilliermondii and Candida ethanolica against bacterial wilt disease of tomato. J Phytol 3:01-10

Nilsson RH, Kristiansson E, Ryberg M, Hallenberg N, Larsson KH (2008) Intraspecific ITS variability in the kingdom fungi as expressed in the international sequence databases and its implications for molecular species identification. Evol Bioinformatics Online 4:193-201

Ochora J, Stock W, Linder H, Newton L (2001) Symbiotic seed germination in twelve Kenyan orchid species. Syst Geogr Plants 71:585596

Otero JT, Flanagan NS, Herre EA, Ackerman JD, Bayman P (2007) Widespread mycorrhizal specificity correlates to mycorrhizal function in the neotropical, epiphytic orchid Ionopsis utricularioides (Orchidaceae). Am J Bot 94:1944-1950

Pinto AJ, Raskin L (2012) PCR biases distort bacterial and archaeal community structure in pyrosequencing datasets. PLoS ONE 7: e43093 
Pridgeon A, Cribb P, Chase M, Rasmussen F (2005) Genera orchidacearum: epidendroideae (Part one). Oxford University Press, Oxford

Rao CR (1982) Gini-Simpson index of diversity: a characterization, generalization and applications. Util Math 21:273-282

Rasmussen HN (1995) Terrestrial orchids: from seed to mycotrophic plant. Cambridge University Press, New York

Rinaldi A, Comandini O, Kuyper TW (2008) Ectomycorrhizal fungal diversity: seperating the wheat from the chaff. Fungal Divers 33:1-45

Roche SA, Carter RJ, Peakall R, Smith LM, Whitehead MR, Linde CC (2010) A narrow group of monophyletic Tulasnella (Tulasnellaceae) symbiont lineages are associated with multiple species of Chiloglottis (Orchidaceae): implications for orchid diversity. Am J Bot 97:1313-1327

Rosselló-Mora R, Amann R (2001) The species concept for prokaryotes. FEMS Microbiol Rev 25:39-67

Runkle E, Wang Y, Blanchard M, Lopez R (2007) Growing the best Phalaenopsis, part 1: an introduction to potted Phalaenopsis orchids. Orchids 76:24-28

Schenck S, Kendrick W, Pramer D (1977) A new nematode-trapping hyphomycete and a reevaluation of Dactylaria and Arthrobotrys. Can J Bot 55:977-985

Schloss PD, Gevers D, Westcott SL (2011) Reducing the effects of PCR amplification and sequencing artifacts on 16S rRNA-based studies. PLoS ONE 6:e27310

Schoch CL, Seifert KA, Huhndorf S, Robert V, Spouge JL, Levesque CA, Chen W, Bolchacova E, Voigt K, Crous PW, Miller AN, Wingfield MJ, Aime MC, An KD, Bai FY, Barreto RW, Begerow D, Bergeron MJ, Blackwell M, Boekhout T, Bogale M, Boonyuen N, Burgaz AR, Buyck B, Cai L, Cai Q, Cardinali G, Chaverri P, Coppins BJ, Crespo A, Cubas P, Cummings C, Damm U, de Beer ZW, de Hoog GS, Del-Prado R, Dentinger B, Dieguez-Uribeondo J, Divakar PK, Douglas B, Duenas M, Duong TA, Eberhardt U, Edwards JE, Elshahed MS, Fliegerova K, Furtado M, Garcia MA, Ge ZW, Griffith GW, Griffiths K, Groenewald JZ, Groenewald M, Grube M, Gryzenhout M, Guo LD, Hagen F, Hambleton S, Hamelin RC, Hansen K, Harrold P, Heller G, Herrera C, Hirayama K, Hirooka Y, Ho HM, Hoffmann K, Hofstetter V, Hognabba F, Hollingsworth PM, Hong SB, Hosaka K, Houbraken J, Hughes K, Huhtinen S, Hyde KD, James T, Johnson EM, Johnson JE, Johnston PR, Jones EBG, Kelly LJ, Kirk PM, Knapp DG, Koljalg U, Kovacs GM, Kurtzman CP, Landvik S, Leavitt SD, Liggenstoffer AS, Liimatainen K, Lombard L, Luangsa-ard JJ, Lumbsch HT, Maganti H, Maharachchikumbura SSN, Martin MP, May TW, McTaggart AR, Methven AS, Meyer W, Moncalvo JM, Mongkolsamrit S, Nagy LG, Nilsson RH, Niskanen T, Nyilasi I, Okada G, Okane I, Olariaga I, Otte J, Papp T, Park D, Petkovits T, Pino-Bodas R, Quaedvlieg W, Raja HA, Redecker D, Rintoul TL, Ruibal C, Sarmiento-Ramirez JM, Schmitt I, Schussler A, Shearer C, Sotome K, Stefani FOP, Stenroos S, Stielow B, Stockinger H, Suetrong S, Suh SO, Sung GH, Suzuki M, Tanaka K, Tedersoo L, Telleria MT, Tretter E, Untereiner WA, Urbina H, Vagvolgyi C,
Vialle A, Vu TD, Walther G, Wang QM, Wang Y, Weir BS, Weiss M, White MM, Xu J, Yahr R, Yang ZL, Yurkov A, Zamora JC, Zhang N, Zhuang WY, Schindel D (2012) From the cover: nuclear ribosomal internal transcribed spacer (ITS) region as a universal DNA barcode marker for Fungi. Proc Natl Acad Sci 109:6241-6246

Schulz B, Boyle C (2005) The endophytic continuum. Mycol Res 109: 661-686

Seena S, Pascoal C, Marvanová L, Cássio F (2010) DNA barcoding of fungi: a case study using ITS sequences for identifying aquatic hyphomycete species. Fungal Divers 44:77-87

Shannon C (1948) A mathematical theory of communication. AT\&T Tech J 27:623-656

Smith SE, Read DJ (2008) Mycorrhizal symbiosis, 3rd edn. Academic, Amsterdam

Stockinger H, Krüger M, Schüßler A (2010) DNA barcoding of arbuscular mycorrhizal fungi. New Phytol 187:461-474

Suárez JP, Weiß M, Abele A, Garnica S, Oberwinkler F, Kottke I (2006) Diverse tulasnelloid fungi form mycorrhizas with epiphytic orchids in an Andean cloud forest. Mycol Res 110:1257-1270

Tringe SG, Hugenholtz P (2008) A renaissance for the pioneering 16S rRNA gene. Curr Opin Microbiol 11:442-446

Vega FE, Posada F, Peterson SW, Gianfagna TJ, Chaves F (2006) Penicillium species endophytic in coffee plants and ochratoxin A production. Mycologia 98:31-42

Vilgalys R, Hester M (1990) Rapid genetic identification and mapping of enzymatically amplified ribosomal DNA from several Cryptococcus species. J Bacteriol 172:4238-4246

Wakelin S, Gupta VV, Harvey P, Ryder M (2007) The effect of Penicillium fungi on plant growth and phosphorus mobilization in neutral to alkaline soils from southern Australia. Can J Microbiol 53: $106-115$

Wang Y-T (2004) Flourishing market for potted orchids. FlowerTech 7:2-

Wey G (1988) Occurrence and investigation of important diseases on Phalaenopsis in Taiwan. Rep Taiwan Sugar Res Inst 122:31-41

Wu Z, Wang X-R, Blomquist G (2002) Evaluation of PCR primers and PCR conditions for specific detection of common airborne fungi. J Environ Monitor 4:377-382

Wu P-H, Huang D-D, Chang DCN (2011) Mycorrhizal symbiosis enhances Phalaenopsis orchid's growth and resistence to Erwinia chrysanthemi. Afr J Biotechnol 10:10095-10100

Yang Y, Cai L, Yu Z, Liu Z, Hyde KD (2011) Colletotrichum species on Orchidaceae in southwest China. Cryptogam Mycol 32:229-253

Zelmer CD, Cuthbertson L, Currah RS (1996) Fungi associated with terrestrial orchid mycorrhizas, seeds and protocorms. Mycoscience 37:439-448

Zeng QY, Rasmuson-Lestander Å, Wang XR (2004) Extensive set of mitochondrial LSU rDNA-based oligonucleotide probes for the detection of common airborne fungi. FEMS Microb Lett 237:79-87

Zhang X, Andrews JH (1993) Evidence for growth of Sporothrix schenckii on dead but not on living Sphagnum moss. Mycopathologia 123:87-94 